\title{
ESTRATÉGIAS DE CONTROLE PREDITIVO APLICADAS AO PROCESSO DE HIDROTRATAMENTO DE DIESEL
}

\author{
J. I. S. da SILVA ${ }^{1}$, E. C. BISCAIA Jr. ${ }^{1}$, M. B. SOUZA Jr. ${ }^{2}$ e A. R. SECCHI ${ }^{1}$ \\ ${ }^{1}$ Universidade Federal do Rio de Janeiro, Programa de Engenharia Química/COPPE \\ ${ }^{2}$ Universidade Federal do Rio de Janeiro, Escola de Química \\ E-mail para contato: josesilva@ peq.coppe.ufrj.br
}

\begin{abstract}
RESUMO - A presença de contaminantes no óleo diesel é severamente controlada. Estes componentes afetam as propriedades do diesel, causando impactos nas emissões de poluentes e comprometendo sua comercialização. A legislação impõe o uso de diesel mais puro, como o diesel S10. Este trabalho aborda a modelagem fenomenológica e controle avançado do processo de hidrotratamento de diesel, tendo como base mecanismos existentes na literatura. $\mathrm{Na}$ representação do processo foi considerado um modelo dinâmico trifásico de um reator de leito gotejante. Uma estratégia de controle preditivo baseada em modelo (MPC) foi implementada com o objetivo de controlar a concentração de enxofre na saída do reator, manipulando a vazão de líquido na carga, na presença de distúrbios na vazão de hidrogênio. Observou-se que a estratégia de controle levou o teor de contaminante para dentro da faixa de especificação do diesel S10.
\end{abstract}

\section{INTRODUÇÃO}

Nos últimos anos, problemas ambientais se tornaram questão importante nas discussões em todo o mundo, elevando os anseios por uma legislação firme no controle de poluições, em especial quando se trata de emissões oriundas do uso de produtos derivados do petróleo. O Conselho Nacional do Meio Ambiente criou, em 1986, o Programa de Controle de Poluição do Ar por Veículos Automotores, o qual fixa limites de emissões e estabelece o desenvolvimento de tecnologias para os veículos em circulação nacional (Ferreira, 2011). A legislação atual indica que óleo diesel mais puro deve substituir os mais poluentes. O diesel que atende a estas recomendações é o Diesel S10, que contém no máximo 10 ppm em termos de enxofre em sua composição. A produção deste combustível requer o uso intensivo de unidades de hidrotratamento (HDT), com a necessidade de catalisadores de elevada atividade e condições operacionais severas (Krause, 2011).

O HDT é um processo no qual a carga, praticamente qualquer fração do petróleo, reage com hidrogênio em elevadas pressões e temperaturas, na presença de um catalisador heterogêneo. O HDT de diesel é feito com o uso de um reator de leito gotejante (Trickle Bed Reactor - TBR) que permite a redução do teor de enxofre e de outros contaminantes. Desta forma, as correntes de óleo diesel a ser hidrotratado e de hidrogênio fluem através de um leito fixo de catalisador (Murali et al., 2007).

Segundo Ferreira (2011) e Aye e Zhang (2005), modelos cinéticos são fundamentais para que se 


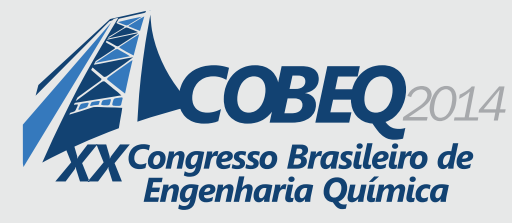

tenha um bom desempenho da unidade de HDT, e que a construção de modelos lida com obstáculos que dificultam a identificação e a quantificação dos compostos organossulfurados e organonitrogenados contidos no óleo. Na Figura 1, é apresentado um esquema típico de um TBR empregado neste tipo de processo, em que normalmente são introduzidas duas correntes de processo no reator: uma contendo o óleo e outra o gás hidrogênio, gerando gases (como o gás sulfídrico) e o hidrocarboneto com o grau de pureza desejado.

Para atender às especificações existentes que determinam o teor de contaminantes no diesel, o processo de HDT precisa ser controlado e otimizado, visando a maior rentabilidade da operação. O Controle Preditivo (MPC ou Model Predictive Control) é considerado a maneira mais geral de colocar o problema de controle no domínio do tempo. As diversas aplicações dos controladores MPC os habilitam como sistemas de controle eficientes, capazes de operar por longos períodos de tempo com pouca intervenção (Ogunnaike e Ray, 1994).

O MPC designa métodos de controle em que há uso explícito de um modelo para predizer a saída do processo em instantes de tempo futuros (horizonte). Também há o cálculo de uma sequência de controle que minimiza uma função objetivo e, finalmente, uma estratégia de horizonte descendente (com atualização feedback, em que o valor medido é realimentado). No controle preditivo, com base em informações passadas de $u$ (entradas) e $y$ (saídas) e em uma projeção para as ações de controle futuras, a saída de um modelo do processo é predita ao longo de um horizonte de predição P. Uma sequência de setpoints futuros (também chamada de trajetória de referência) é gerada. Uma função objetivo quadrática apropriada de erros futuros e ações de controle é minimizada para fornecer uma sequência sugerida de ações de controle futuras (ver Figura 2). Neste tipo de controle, apenas a primeira ação de controle é implementada (horizonte descendente).

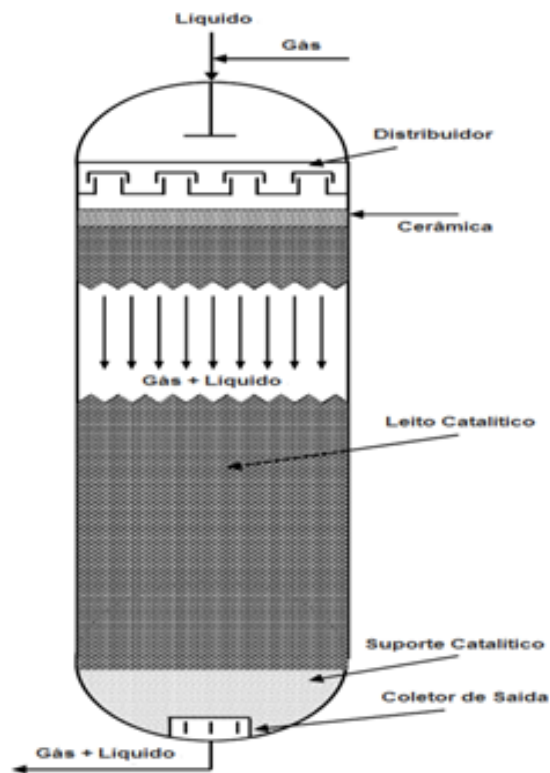

Figura 1 - Diagrama esquemático de um TBR com operação em cocorrente.

Fonte: Adaptado de Mederos e Ancheyta (2007).

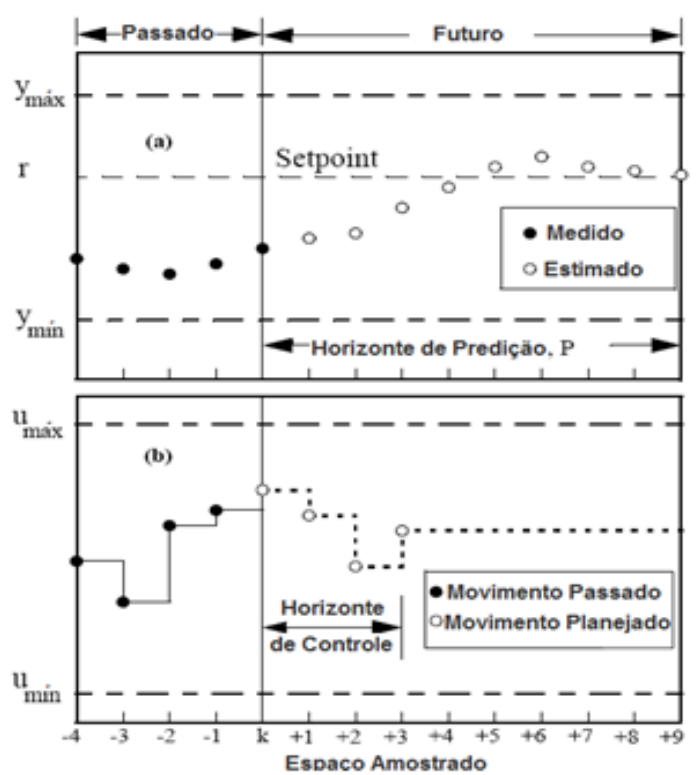

Figura 2 -Elementos do MPC: (a) Saída predita, horizonte de predição P: (b) Ações de controle. Fonte: Adaptado de Ogunnaike e Ray (1994). 
O objetivo deste trabalho foi propor uma estratégia de controle preditivo ao processo de HDT de diesel, visando controlar o teor de enxofre na saída do TBR, buscando obter o diesel na especificação S10, manipulando a vazão de líquido na entrada do reator.

\section{METODOLOGIA}

A modelagem do processo foi abordada baseando-se nos estudos de Korsten e Hoffmann (1996) e de Mederos e Ancheyta (2007), os quais pesquisaram sobre o processo de HDT de diesel, considerando um sistema trifásico operando em escala comercial em escoamento concorrente (Equações 3 a 9).

As frações de petróleo contêm uma grande quantidade de componentes organossulfurados, nitrogenados, dentre outros. Contudo a principal reação que ocorre no processo HDT de diesel é a reação de hidrodessulfurização (HDS). Neste trabalho foi considerada apenas a reação de HDS. O modelo cinético para a reação de HDS é do tipo Langmuir-Hinshelwood (Equações 1 e 2).

$r_{H D S}=\frac{k_{H D S}\left(C_{S}^{S}\right)\left(C_{H 2}^{S}\right)^{0,45}}{\left(1+k_{H 2 S}\left(C_{H 2}^{S}\right)\right)^{2}}$

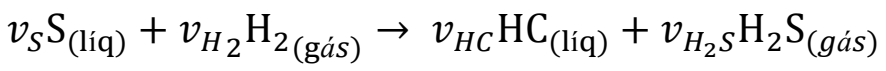

As equações do balanço são mostradas a seguir, em que $r_{H D S}$ é a taxa da reação, $k_{H D S}$ é constante aparente da reação, $C_{i}^{S}$ é a concentração da espécie $i$ no sólido, $k_{H 2 S}$ é a constante de equilíbrio de adsorção para o $\mathrm{H}_{2} \mathrm{~S}, v_{i}$ é o coeficiente estequiométrico, $\mathrm{S}$ são os compostos organossulfurados e $\mathrm{HC}$ é o hidrocarboneto.

\subsection{Balanço de massa}

Fase gasosa:

$$
\varepsilon_{G} \frac{\partial p_{i}}{\partial t}=-u_{G} \frac{\partial p_{i}}{\partial z}-R T_{G} k_{i}^{L} a_{L}\left(\frac{p_{i}}{\mathcal{H}_{i}}-C_{i}^{L}\right) \quad i=\mathrm{H}_{2} \text { e } \mathrm{H}_{2} \mathrm{~S}
$$

Fase líquida:

$$
\begin{array}{cc}
\varepsilon_{L} \frac{\partial C_{i}^{L}}{\partial t}=-u_{L} \frac{\partial C_{i}^{L}}{\partial z}+\varepsilon_{L} D_{a}^{L} \frac{\partial^{2} C_{i}^{L}}{\partial z^{2}}+k_{i}^{L} a_{L}\left(\frac{p_{i}}{\mathcal{H}_{i}}-C_{i}^{L}\right)-k_{i}^{S} a_{S}\left(C_{i}^{L}-C_{i}^{S}\right) & i=\mathrm{H}_{2} \mathrm{e}_{2} \mathrm{~S} \\
\varepsilon_{L} \frac{\partial C_{i}^{L}}{\partial t}=-u_{L} \frac{\partial C_{i}^{L}}{\partial z}+\varepsilon_{L} D_{a}^{L} \frac{\partial^{2} C_{i}^{L}}{\partial z^{2}}-k_{i}^{S} a_{S}\left(C_{i}^{L}-C_{i}^{S}\right) & i=\mathrm{HC} \text { e } \mathrm{S}
\end{array}
$$

Fase sólida:

$$
\varepsilon_{p}(1-\epsilon) \frac{\partial C_{i}^{S}}{\partial t}=k_{i}^{S} a_{S}\left(C_{i}^{L}-C_{i}^{S}\right) \pm \rho_{B} \zeta \eta_{H D S} r_{H D S} \quad i=\mathrm{H}_{2}, \mathrm{H}_{2} \mathrm{~S}, \mathrm{HC} \text { e } \mathrm{S}
$$


Na Equação (6), o sinal "-" significa que é para os reagentes e o sinal "+" para os produtos; $\varepsilon_{j}$ é o holdup da fase $j$, $u$ é a velocidade, $T$ é a temperatura, $z$ é a posição axial no TBR, $p_{i}$ é a pressão parcial de $i, \mathcal{H}_{i}$ é a constante de Henry, $C_{\mathrm{i}}$ é a concentração da espécie $i, a_{L}$ e $a_{S}$ são as áreas específicas nas interfaces, $D_{a}^{L}$ é o coeficiente de dispersão axial (mássico), $k_{i}^{j}$ é o coeficiente de transferência de massa da espécie $i$ na interface $j, \in$ é fração de vazios do leito, $\varepsilon_{p}$ é a porosidade da partícula de catalisador, $\rho_{B}$ é a densidade $b u l k, \zeta$ é a fração de volume do leito diluído, $\eta_{H D S}$ é fator de eficiência catalítica.

\subsection{Balanço de energia}

Fase gasosa:

$\varepsilon_{G} \rho_{G} C_{P G} \frac{\partial T_{G}}{\partial t}=-u_{G} \rho_{G} C_{P G} \frac{\partial T_{G}}{\partial z}-h_{G L} a_{L}\left(T_{G}-T_{L}\right)$

Fase líquida:

$\varepsilon_{L} \rho_{L} C_{P L} \frac{\partial T_{L}}{\partial t}=-u_{L} \rho_{L} C_{P L} \frac{\partial T_{L}}{\partial z}+h_{G L} a_{L}\left(T_{G}-T_{L}\right)-h_{L S} a_{S}\left(T_{L}-T_{S}\right)$

Fase sólida:

$$
(1-\epsilon) \rho_{S} C_{P S} \frac{\partial T_{S}}{\partial t}=h_{L S} a_{S}\left(T_{L}-T_{S}\right)+\rho_{B} \eta_{H D S} r_{H D S}\left(-\Delta H_{\mathrm{HDS}}\right)
$$

Em que $\rho_{j}$ é a densidade da fase $j, h_{G L}$ e $h_{L S}$ são os coeficientes de troca de calor na interface gás-líquido e líquido-sólido, respectivamente, $\Delta H_{\mathrm{HDS}}$ é o calor da reação.

\subsection{Integração numérica}

O sistema de equações diferenciais parciais, descrevendo a transferência de calor e massa no reator, foi transformado em um conjunto de equações diferenciais ordinárias de primeira ordem, utilizando o método das diferenças finitas centrais na direção axial. Utilizou-se o EMSO (Environment for Modeling, Simulation and Optimization) como ambiente para esta integração, fazendo-se uso dos algorítmos sundials e dasslc. As condições de operação, os parâmetros e as correlações utilizadas para o cálculo de propriedades do sistema foram retirado dos trabalhos de Korsten e Hoffmann (1996), Mederos e Ancheyta (2007), Jiménez et al. (2007) e Mederos et al. (2012).

\subsection{Diagrama de bloco no Simulink e criação da s-function}

A estratégia de controle foi implementada no Simulink (MATLAB ${ }^{\circledR}$ R2010a). Criou-se uma interface EMSO-MATLAB para simular a planta no MATLAB. A planta foi colocada no Simulink com o uso da interface. Na Figura 3, pode ser visualizado o esquema proposto, em que foi criada uma $s$-function (a qual permite chamar a planta real no EMSO), e que no bloco em azul foi configurada esta função (com três variáveis de entrada, uma de saída, e tempo de amostragem de 5 segundos). 


\subsection{Identificação do sistema}

Testes foram realizados com o objetivo de obter dados para identificar o sistema, e foram aplicadas perturbações degrau na entrada da planta (vazão de líquido) em torno do estado estacionário. Os dados de entrada e saída foram levado até o ident (System Identification Toolbox) no MATLAB.

\subsection{Implementação e sintonia do MPC}

O bloco MPC foi adicionado ao diagrama de bloco no Simulink, como mostrado na Figura 3.

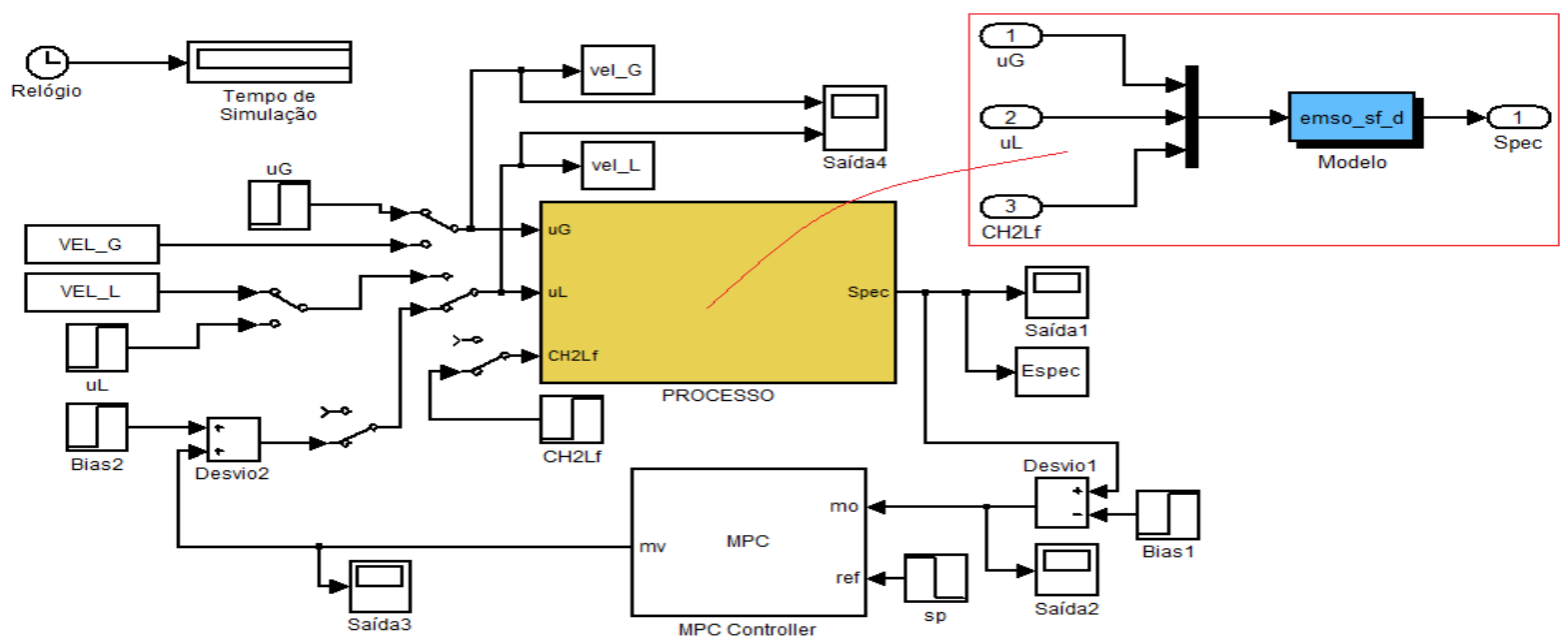

Figura 3 - Diagrama de bloco da planta com a inserção do MPC.

\section{RESULTADOS E DISCUSSÃO}

A identificação do sistema com o uso do toolbox ident do MATLAB e as perturbações da Figura 4 geraram um modelo simplificado da planta de terceira ordem com tempo morto e resposta inversa, Figura 5. Nessa figura a linha preta (tracejada) é a resposta da planta simulada e a linha verde (contínua) é a resposta do modelo identificado (com coeficiente de correlação dos dados ajustados igual a 93,37).

A sintonia do controlador MPC foi feita seguindo critérios estabelecidos na literatura (Seborg (1989) e Ogunnaike e Ray (1994)), bem como da análise da resposta ao degrau unitário na saída.

O controlador projetado fez com que a resposta seguisse o setpoint, atingindo a especificação do Diesel S10 para alguns cenários testados. Contudo, em outros cenários analisados, percebe-se que o alvo não foi alcançado. Na Figura 6, verifica-se que a especificação foi atendida, com o horizonte de predição (P) igual a 200 e horizonte de controle (M) igual a 50, e para P igual a 100 e M igual a 50, respectivamente, com tempo de amostragem (ta) igual a 5 segundos e peso da variável controlada de $10^{-7}$. Também na Figuras 6, $\mathrm{P}$ e $\mathrm{M}$ foram reduzidos, percebe-se que a resposta tende a ficar fora da especificação, e que quando o peso foi reduzido, a resposta não consegue se aproximar do setpoint (ver as curvas azul claro, preta e amarela da Figura 6). 


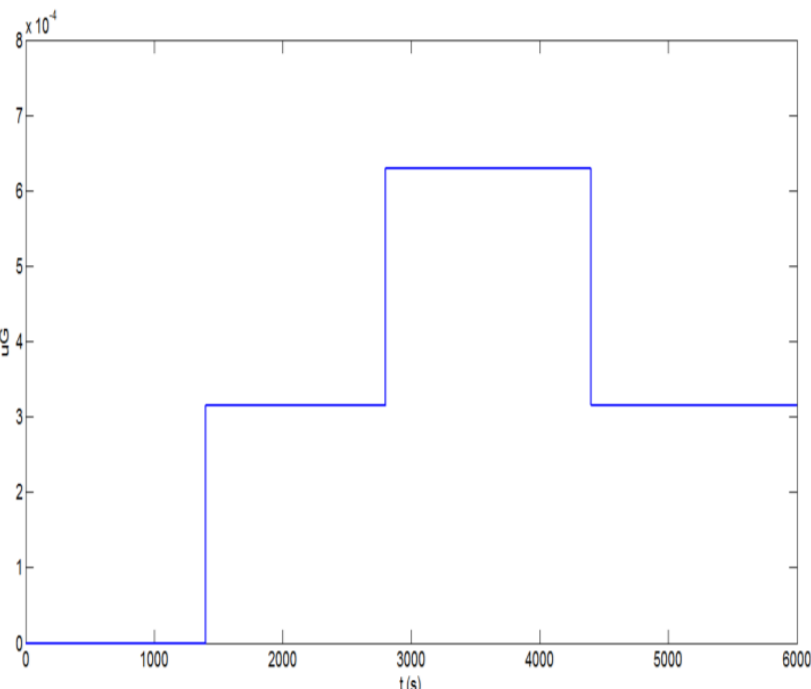

Figura 4 - Perturbações aplicadas na vazão de líquido.

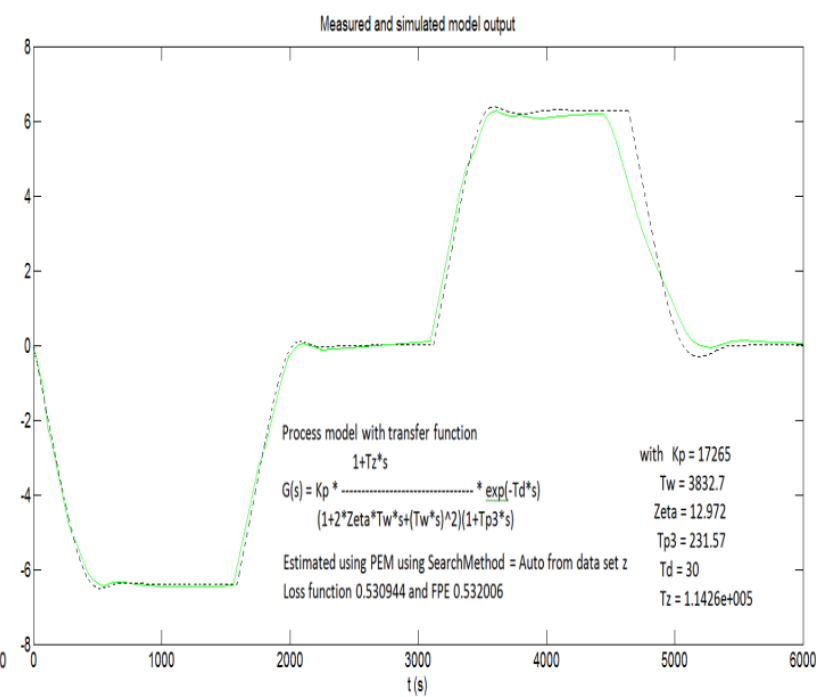

Figura 5 - Identificação do sistema: função de transferência do processo.

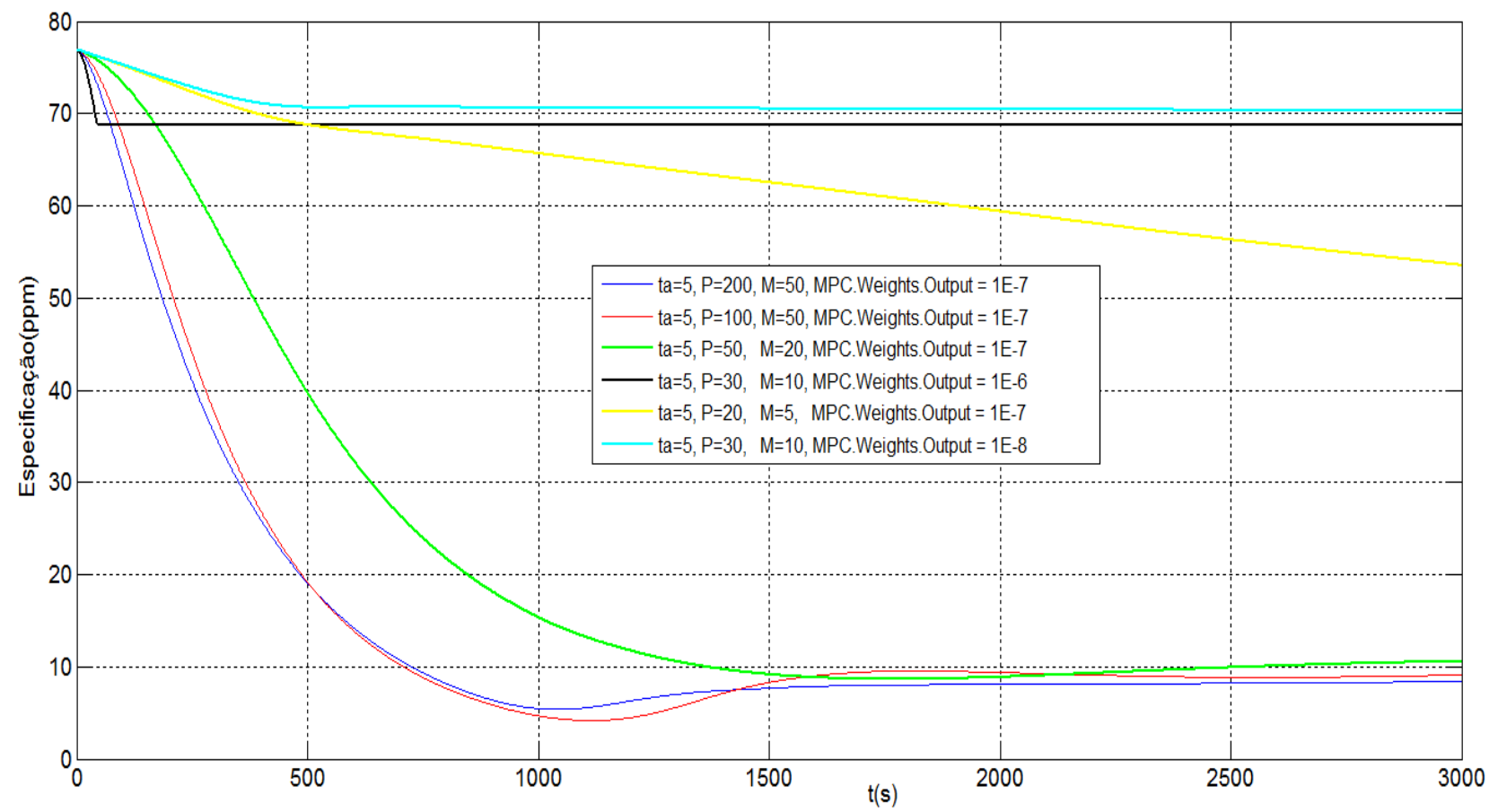

Figura 6 - Respostas do MPC nos horizontes dados.

Foi feita perturbação (-20\%) na vazão de hidrogênio no tempo de 2000 segundos de simulação, contudo a resposta não mostrou sensibilidade significativa, como é mostrado na Figura 7a. A ação na variável manipulada pode ser observada na Figura 7b. 

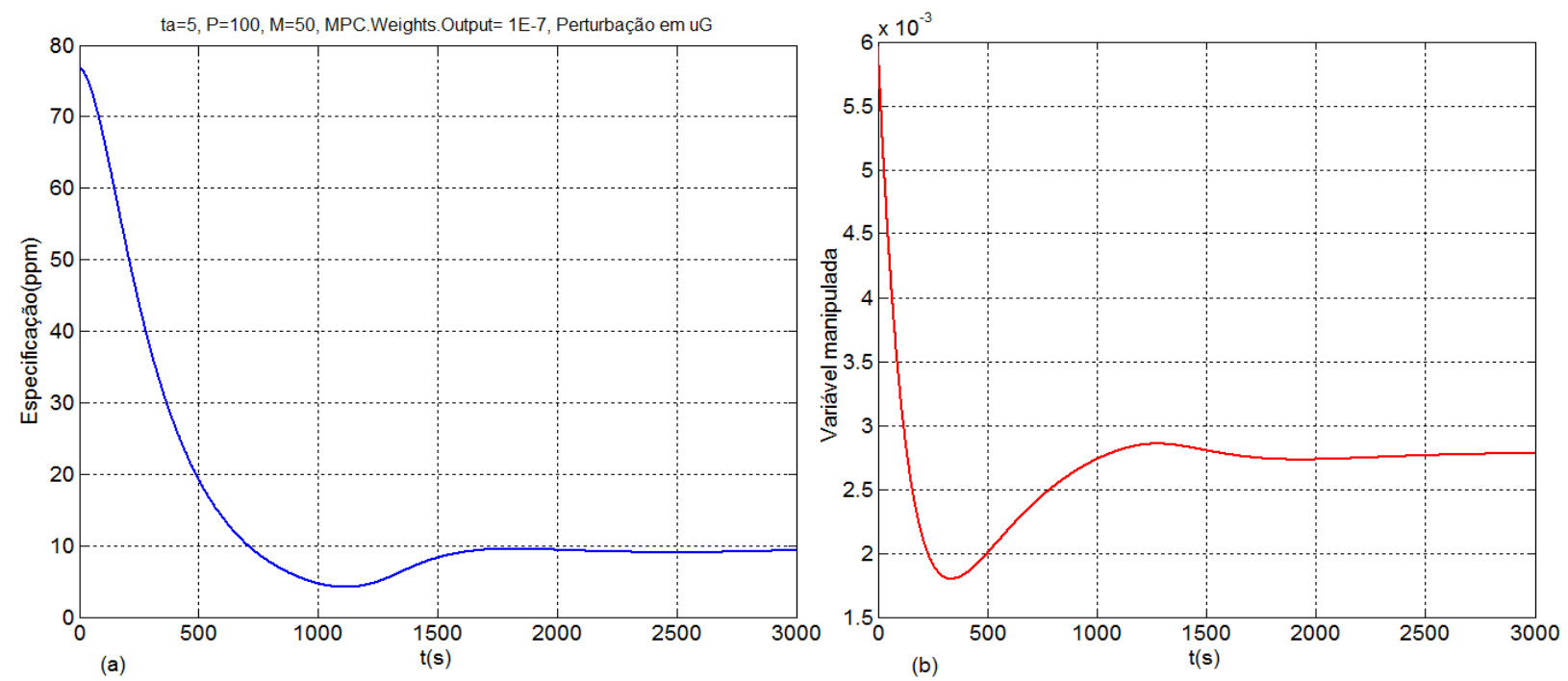

Figura 7 - Resposta: (a) Perturbação em -20\% na vazão de $\mathrm{H}_{2}$; (b) Ações na variável manipulada, da qual chegou-se à especificação.

Perturbações no setpoint foram analisadas, chegando-se à especificação. Nas Figuras 8a e 8 b, pode ser verificado o comportamento da resposta frente à perturbação.
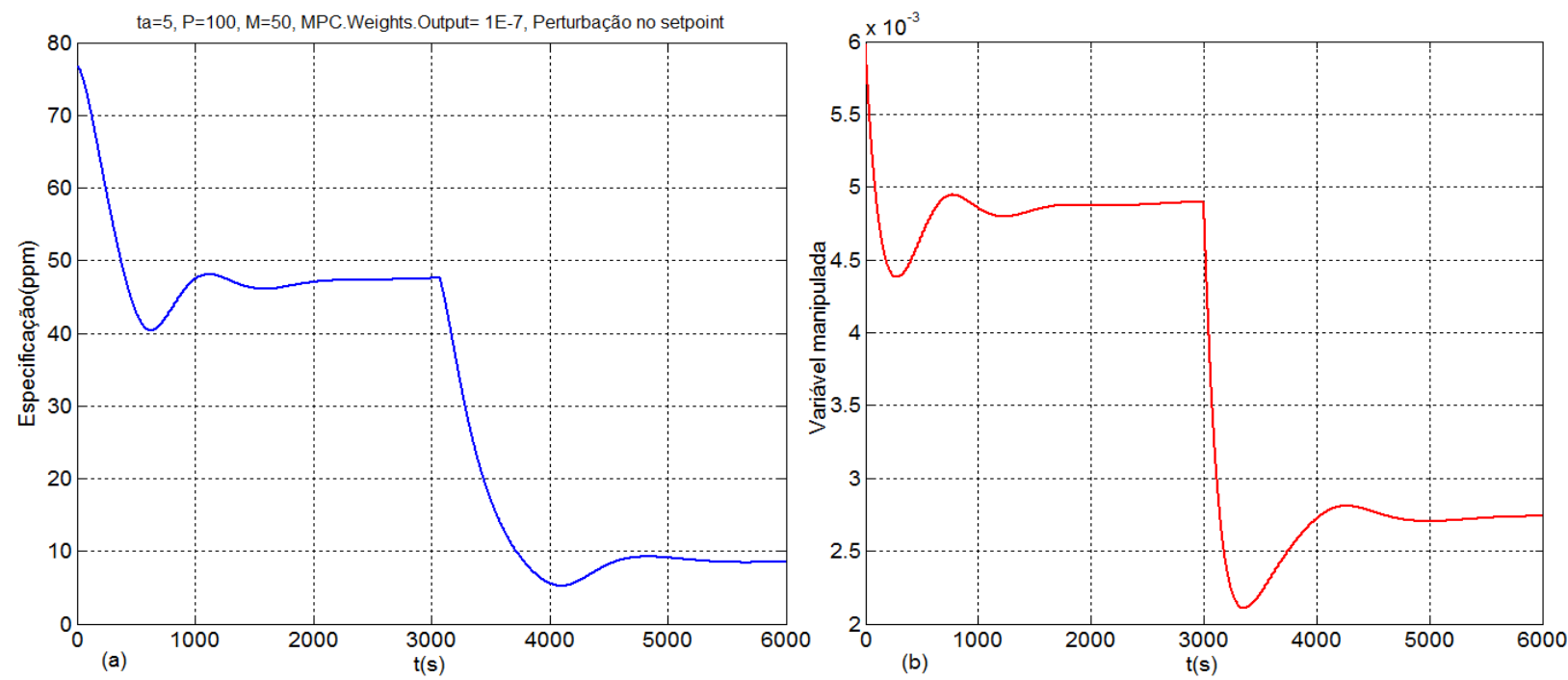

Figura 8 - Resposta: (a) Perturbação, setpoint em 50 ppm, e depois de estabilizado pertubado para 10 ppm; (b) Variável manipulada.

\section{CONCLUSÕES}

Neste trabalho, implementou-se no simulador EMSO um modelo de reator em leito gotejante para o processo de hidrotratamento de diesel e uma plataforma no SIMULINK/MATLAB para estudos de controle avançado, especificamente, o controle 
preditivo baseado em modelo.

Estudos preliminares do comportamento servo e regulador de uma malha SISO de controle da composição da saída pela manipulação da vazão da carga mostraram a capacidade do controlador em atender a esses objetivos.

A estrutura implementada permite facilmente que estudos futuros mais aprofundados sejam realizados, nos quais aspectos relacionados à natureza multivariável e não linear do problema e a presença de restrições imponham desafios mais rigorosos ao controlador. Outro desenvolvimento futuro diz respeito à validação do modelo implementado com dados reais de planta.

\section{REFERÊNCIAS}

AYE, M. M. S.; ZHANG, N. A novel methodology in transforming bulk properties of refining streams into molecular information. Chem. Eng. Science, v. 60, p. 6702-6717, 2005.

FERREIRA, A. S. Busca Heurística e Inferência de Parâmetros Cinéticos de Reações de Hidrotratamento de Óleo Diesel a Partir de Dados Experimentais Escassos. PPGEQ/UFSCar, São Carlos, 2011.

JIMENEZ, F.; OJEDA, K.; SANCHEZ, E.; KAFAROV, V.; MACIEL FILHO, R. Modeling of trickle bed reactor for hydrotreating of vacuum gas oils: effect of kinetic type on reactor modeling. Computer Aided Chem. Eng., v. 24, p. 515-520, 2007.

KORSTEN, H.; HOFFMANN, U. Three-Phase reactor model for hydrotreating in pilot tricke-bed reactors. AIChE J., v. 42, p. 1350, 1996.

KRAUSE, B. B. Simulação da Desativação de Catalisadores $\mathrm{NiMo} / \mathrm{Al}_{2} \mathrm{O}_{3} \mathrm{em}$ uma Unidade Industrial de Hidrotratamento. PEQ/COPPE/UFRJ, Rio de Janeiro, 2011.

MEDEROS, F. S.; ANCHEYTA, J.; ELIZALDE, I. Dynamic modeling and simulation of hydrotreating of gas oil obtained from heavy crude oil. Appl. Cat. A: General, v. 425-426, p. 13-27, 2012.

MEDEROS, F. S.; ANCHEYTA, J. Mathematical modeling and simulation of hydrotreating reactors: Cocurrent versus countercurrent operations. Appl. Cat. A: General, v. 332, p. 821, 2007.

MURALI, C.; VOOLAPALLI, R. K.; RAVICHANDER, N.; GOKAK, D. T.; CHOUDARY, N. V. Trickle bed reactor model to simulate the performance of commercial diesel hydrotreating unit. Fuel, v. 86, p. 1176-1184, 2007.

OGUnNAIKE, B. A.; RAY, W. H. Process Dynamics, Modeling, and Control. Oxford University Press, NY, 1994.

SEBORG, D. E.; EDGAR, T. F.; MELliCHAMP, D. A. Process Dynamics and Control. New York: J. Wiley, 1989. $717 \mathrm{p}$. 